Gyancity Journal of Electronics and Computer Science

Vol.3, No.2, pp. 32-38, September 2018

ISSN:2446-2918 DOI: 10.21058/gjecs.2018.32005

\title{
LOW-COST COMPUTER VISION ASSISTED OBJECT SEGREGATION ROBOT
}

\author{
Engr. Ahson Ahmed \\ ${ }^{1}$ Student in Electronics \& Biomedical Engineering, \\ IICT, Mehran University of Engineering and \\ Technology, Pakistan \\ Cell: +92 3453587885
}

Email: ahson_ahmed2000@yahoo.com

\begin{abstract}
Low-Cost Computer Vision Assisted Object Segregation Robot" is a research project related to the field of Mechatronics, Image Processing, and Industrial Automation. The aim of the research is to detect three different regular shapes in a single symmetrical object image through $2 D$ low-resolution camera with machine vision techniques and placement of these objects at their designated positions via two-axis robotic arm manipulator. The prototype system and software have been made locally with scrap materials. This project would be useful in Local industries in Pakistan who are focusing on low-cost solutions based on vision-based data acquisitions and Cost Effective automation for products segregation and quality control operations. Local Industries in Pakistan are quite reluctant to adopt latest robotic techniques due to high CAPEX (imported equipment) and a high cost of maintenance. Consequently, the majority of industries in Pakistan are utilizing obsolete sensor-based information for their automation and data acquisition process. The sensor-based system has some drawbacks due to limited information sensing. Majority of complex tasks like inspection of products or segregation of different type of products are mostly done by humans or occasionally with sensor-based approaches. However, said approaches are costly due to of labor cost and also less efficient due to slow operating speeds. Foregoing in view, a locally developed, cost-effective and easily configurable vision-based intelligent robotic solution would be highly beneficial for local industries within Pakistan to streamline their various automation processes which might provide low production cost with strict quality control. Additionally, Pakistan is also an agricultural based economy where major churn of export contain fruits, vegetable, and grains. The countries around the world have strict standards for imported food items. Therefore, the subject project would be beneficial for exporters for intelligent automation of quality inspections, grading of fruits and vegetables according to the quality standards of international markets.
\end{abstract}

Keywords- Computer vision system, Mechatronic systems, Quality Control, Inspection and industrial automation.

\section{INTRODUCTION}

The goal behind the Idea is to develop a Cost-effective simplified Prototype to demonstrate the application Concept for Local Use within the country. As a proof of concept algorithm and prototype is demonstrated in University Lab environment.

The research Prototype is based on following domains:

A) Acquisition of different geometric objects shape via machine vision [2] techniques 
B) Extraction of shapes through Shape Factor, boundary tracing, and edge detection techniques

C) Decision-making algorithms for identification of the geometric structure of objects

D) Low-cost Automation process for object placement at particular container via easily configurable two-axis robotic manipulator with feedback motion limiting I.R Sensors.

The system in the discussion would be able to detect various objects via camera-based machine vision system. Furthermore, a 2-axis robotic manipulator comprising of microcontroller, automotive DC motors and motion limiting I.R feedback sensors have been developed for grabbing and placement of the object at designated locations [3,4]. Initially, the prototype is able to recognize three different objects with placement at specified three locations. Moreover, the capability of the system can be enhanced by incorporating additional profiles of different objects.

\section{RELATED WORK}

Object recognition and segregation is a widely researched topic in the field of cognitive robotics in the world and Developing day by day according to its application in various domains. For Development and application within country, the Cost-effective system with a simplified algorithm with Local Commissioning of the system is the motive behind the development of this prototype with intended area of application. The techniques for object recognition mostly rely on a set of descriptors. In which, a picture is taken from a camera and individual entities in the picture are determined by computing certain values of descriptors. The relations between the 'distances' between these descriptor vectors is what determine the object parameter for shape. A major focus of this project is to apply Matlab Shape Factor Analysis on 2-D Image with improved acquisition with edge detection operator and classifying the shape with Metric Ratio Based Decision Making Algorithm in simplified form.

\section{A) Methodology}

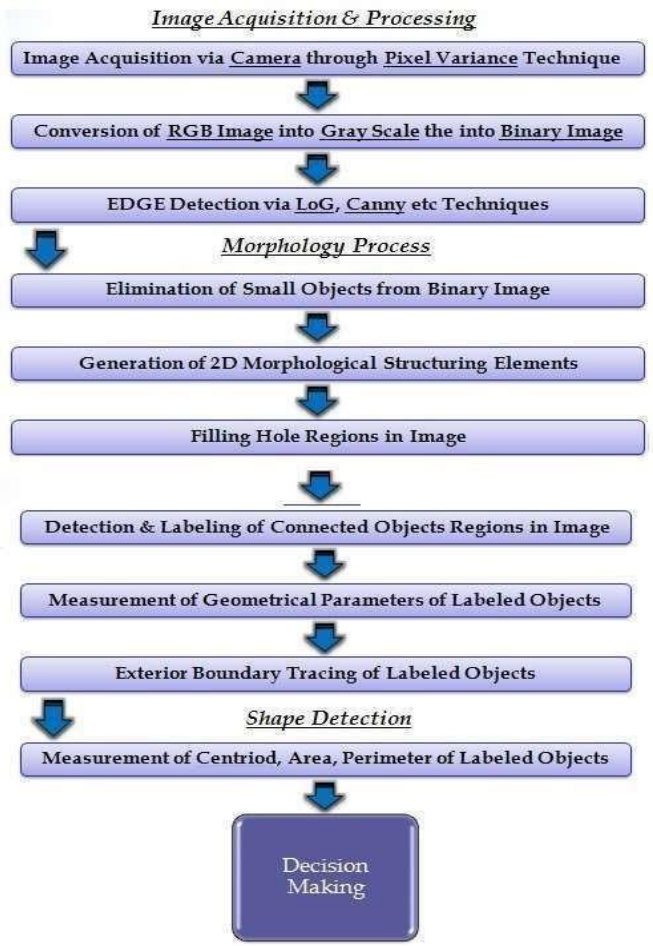

The main section of this research is information acquisition via image sensing. Initially, three types of object shapes i.e. Triangle, Circle, and Square were selected for this research. When an object is placed in 
front of the robotic arm, a snapshot is taken from the camera mounted over aforesaid manipulator. Pixel variance technique is used for video triggering. This technique is based on observing deviation in pixels between multiple video frames. Whenever an object is placed in front of a camera it changes the number of pixels within multiple frames and any pixel changes in the idle frame would trigger the camera to take a snapshot automatically and image of an object having a resolution of 320X 480 jpeg with a black background will be saved accordingly. Due to variant light conditions following steps have been followed for proper edge detection and boundary tracing of objects:

B) Acquisition of RGB (colored) image

C) Conversion of the image from (RGB) to gray image

D) Conversion of gray image to black and white binary image

E) Edge Filtration

F) Boundary Tracing

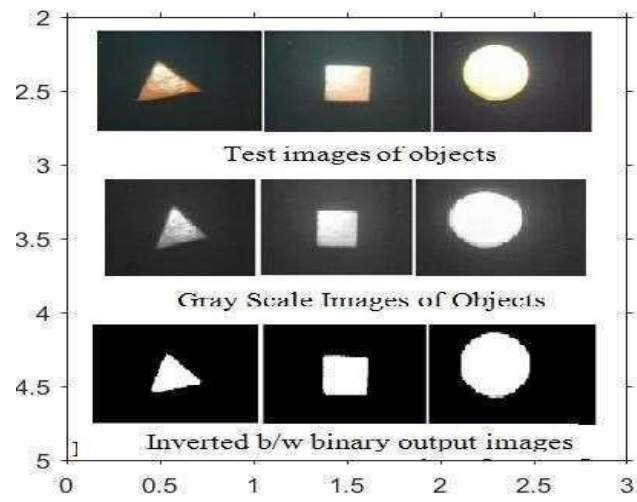

Fig. 1 showing output images of the process

\section{EDGE DETECTION METHOD}

Edge detection is an image processing technique for finding the boundaries of an object(s) within image through discontinuities in brightness. Two edge detection algorithms i.e. Sobel and Canny were used. The results from both filters revealed that canny filter performed well in multiple lighting conditions, resultantly, same will compliment successful boundary tracing. Results of all three shapes are depicted below:
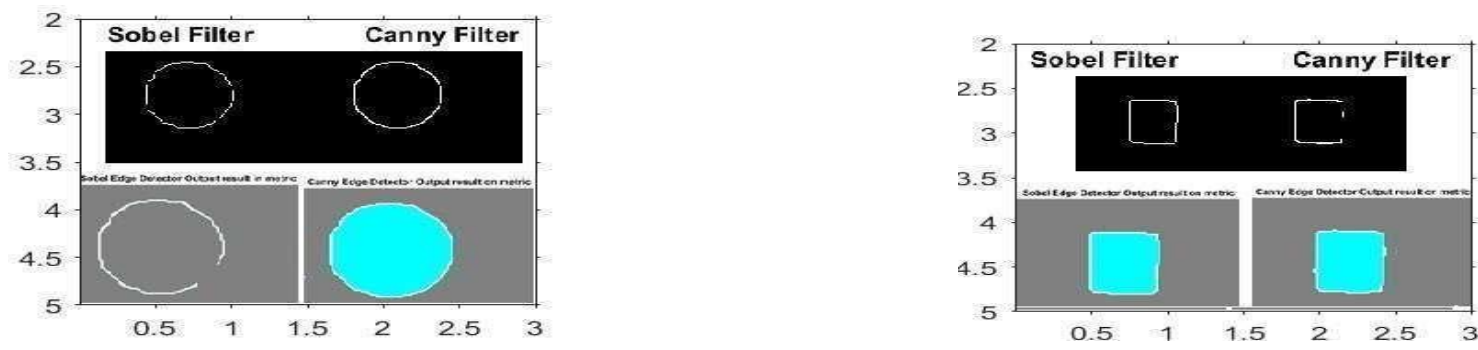

Fig. 2. Edge detection Result of a circular object

Fig. 3. Edge detection Result of square object

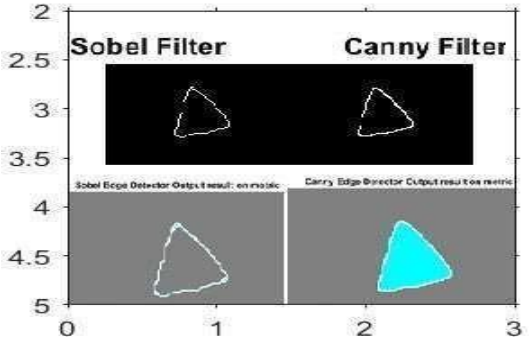

Fig. 4. Edge detection of Triangular object 


\section{A) Morphological Process}

The morphological operations implemented on the image after edge detection for removing unwanted shapes and noise errors from the image to enhance the prominent region i.e. shapes within the image. After processing, noises and unwanted pixels which do not belong to the object of interest were removed. Additionally, holes and gaps were also filled for proper tracing of boundary and area of shapes. Morphology process is shown in Figure 2 5 with a grey background and cyan colored objects.

\section{B) Shape Detection Algorithm [5]}

Shape factor Metric is used which is commonly used in computerized Dynamic Image Analysis (DIA) for particle shape characterization. Shape factor for circularity determination is applied. Shape factor reflects the roundness of an object. A value close to 1 indicates that the object is smooth and round and value close to 0 means that it is elongated and/or rough. Roundness of objects is depicted below:

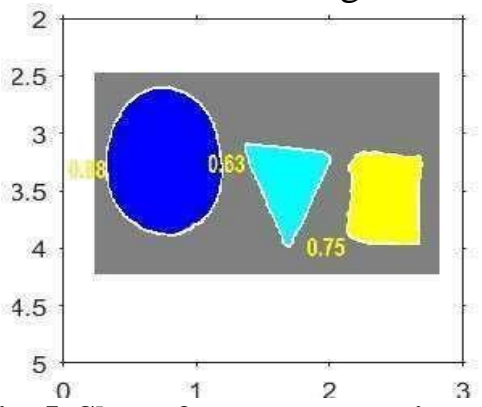

Fig. 5. Shape factor computations Result

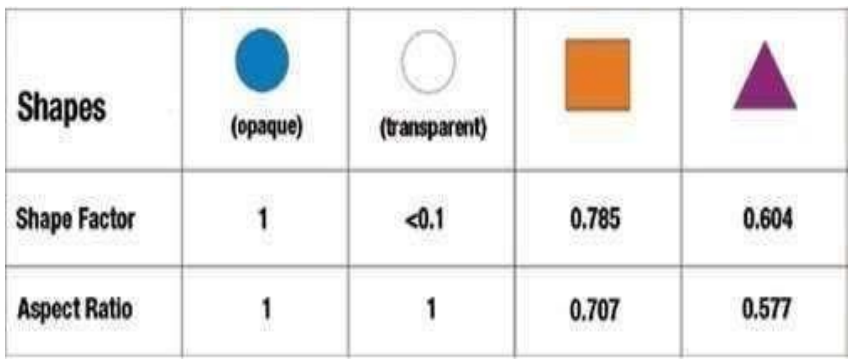

Fig. 6. Shape factor metrics for perfect shapes

The values after computation were compared with the threshold of perfect shapes calculated via shape factor as shown Figure 6. The computed value of any particular shape will be the result of metric of roundness. The circularity of any object is calculated through $\mathrm{R}=4^{*} \mathrm{pi}{ }^{*} \mathrm{~A} / \mathrm{P}^{\wedge} 2$; where $\mathrm{A}$ is an area in pixels, $\mathrm{P}$ is Perimeter. Consequently, the segregating process is managed via comparison of threshold values against each shape as depicted in figure .6. Other shapes can be added after compiling through shape factor metric for thresholds values. Meanwhile, three shapes have been tested successfully and the system is able to make a decision from their respective threshold values depicted in the following flowchart and figure.6.

C) Decision Making Algorithm

Decision-making algorithm works by comparing threshold values of each shape:

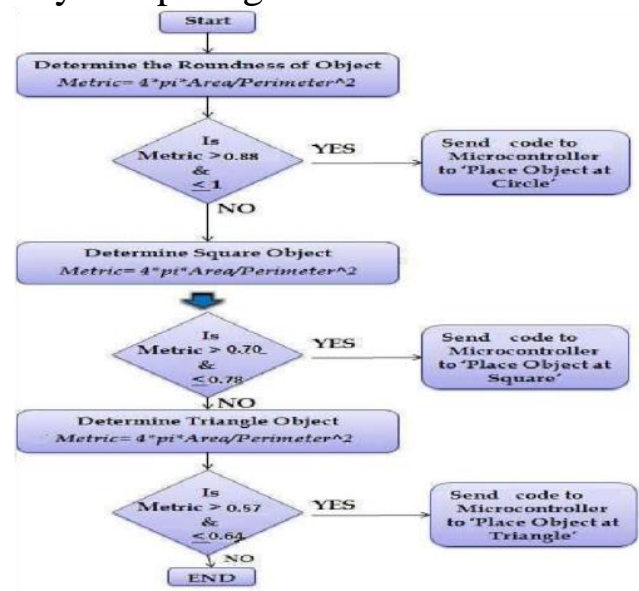

D) Mechanical Manipulator 


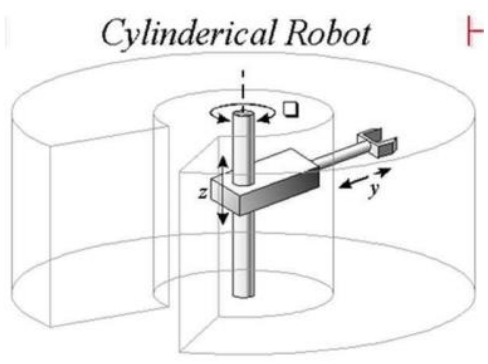

Fig. 7. Manipulator Design

The design of electromechanical prototype is based on the category of service robot commonly used in industries. The electromechanical system contains two-axis robotic arm with motion clearance of 6 meters, a camera, a moveable base and a gripper. Other electrical parts include DC motors with gears [1], relays and infrared sensors for position identification Mechanical slit mechanism is implemented between I.R sensors to limit the horizontal and vertical motion of robotic arm manipulator built with locally available scrap material.

\section{E) Manipulator Controlling Hardware}

Atmel 89C51 microcontroller based embedded system has been developed for controlling the motions of the robotic arm, gripper, and base. Following components were used:

$\begin{array}{ll}\text { a } & \text { AT89C51 Microcontroller } \\ \text { b } & \text { MAX 232 for serial interface } \\ \text { c } & \text { ULN2803 for relay driving } \\ \text { d } & \text { RELAYS for electromechanical switching } \\ \text { e } & \text { ILD74 as optocoupler digital signal isolator } \\ \text { f } & \text { Infrared sensor for motion feedback } \\ \text { g } & \text { Power Supply }\end{array}$

Movement limitation is achieved through sensing positions via I.R sensors. Microcontroller receives an 8-bit binary code via a serial port of the computer and via MAX 232. MCU connectivity is tabulated in Table 1.

Table 1. Ports and Pin Connectivity's

\begin{tabular}{|c|c|c|c|}
\hline S.N & Pins and Ports & Driving ICs & Connected Modules \\
\hline 1. & Port2 pin 0-3 & ULN2803 & Vertical Axis Motor \\
\hline 2. & Port 2 Pin 4-7 & ULN2803 & Horizontal Axis motor \\
\hline 3. & Port 3 Pin 5 & ILD74 & Gripper DC Motor \\
\hline 4. & Port 3 Pin6 & MAX 232 & Serial port connectivity \\
\hline 5. & $0-5 \quad$ Port0 pin & $\begin{array}{c}\text { Feedback IR } \\
\text { Diodes }\end{array}$ & Position Sensing \\
\hline
\end{tabular}

Programming of microcontroller has been done in assembly language. The 8-bit position data is transmitted from the computer for each shape after detection of shape. The code from Matlab is compared with already stored code within microcontroller's memory. When the digital code is matched 
microcontroller executes the routine of specified code and drive the motors of the base, arm, and gripper towards specified positions. Data codes are shown in Table 2.

Table 2. Data Transmission

\begin{tabular}{|c|c|c|}
\hline $\begin{array}{c}\text { Detected } \\
\text { Shape }\end{array}$ & $\begin{array}{c}\text { Data Transmitted } \\
\text { to MCU by Matlab }\end{array}$ & $\begin{array}{c}\text { Manipulator } \\
\text { Moment }\end{array}$ \\
\hline Square & 00100000 & $1^{\text {st }}$ Position \\
\hline Circle & 00010000 & $2^{\text {nd }}$ Position \\
\hline Triangle & 00001000 & $3^{\text {rd }}$ Position \\
\hline
\end{tabular}

The position data through the I.R sensor is received by the MATLAB via MCU for validating the position and task fulfilment. In case of failure or fault, the computer will resend the data again with three attempts if it fails to respond system will automatically move towards halt state and indicate fault via Matlab.

\section{EXPERIMENTAL RESULTS}

In test Case this project was designed to recognize any of the three $2 \mathrm{~d}$ objects (sphere, Square, Triangle) in any colours thus making a total of 3 identifiable objects. During testing, it was found that all 3 objects are successfully identified by shape factor analysis and segregated into their respective positions by the robot also two edge detection filters applied in which canny filter gave good result under test condition shown in figure 2,3,4. Expansion of the code was also successful in specific ambient light conditions for detection of more shapes by comparing the threshold with perfect shapes With GUI implementation The Result and accuracy of detection are shown in Computational Figures $5 \& 6$ above and Performance of shape detection algorithm and Manipulator is shown below.

\begin{tabular}{|l|l|c|c|l|c|}
\hline CASE & IMAGES & OBJECT SHAPE & SHAPE FACTOR & EDGE DETECTOR & RESULT \\
\hline CASE 1 & IMAGE 1 & CIRCULAR & 0.98 & CANNY & DETECTED 98\% \\
\hline CASE 2 & IMAGE 2 & TRIANGULAR & 0.603 & CANNY & DETECTED 63\% \\
\hline CASE 3 & IMAGE 3 & SQUARE & 0.75 & CANNY & DETECTED 75\% \\
\hline
\end{tabular}

Fig. 8. Performance of Shape detection Algorithm

\begin{tabular}{|l|l|l|l|r|}
\hline CASE & OBJECT & PLACEMENT & RESULT + $\%$ & RESULT - $\%$ \\
\hline CASE1 & CIRCULAR & 1ST POSITION & SUCCSESS 80\% & SUCCSESS -20\% \\
\hline CASE2 & TRIANGULAR & 2ND POSITION & SUCCSESS 65\% & SUCCSESS -35\% \\
\hline CASE3 & SQUARE & 3RD POSITION & SUCCSESS 75\% & SUCCESS -25\% \\
\hline
\end{tabular}

Fig. 9. Performance of end effector 


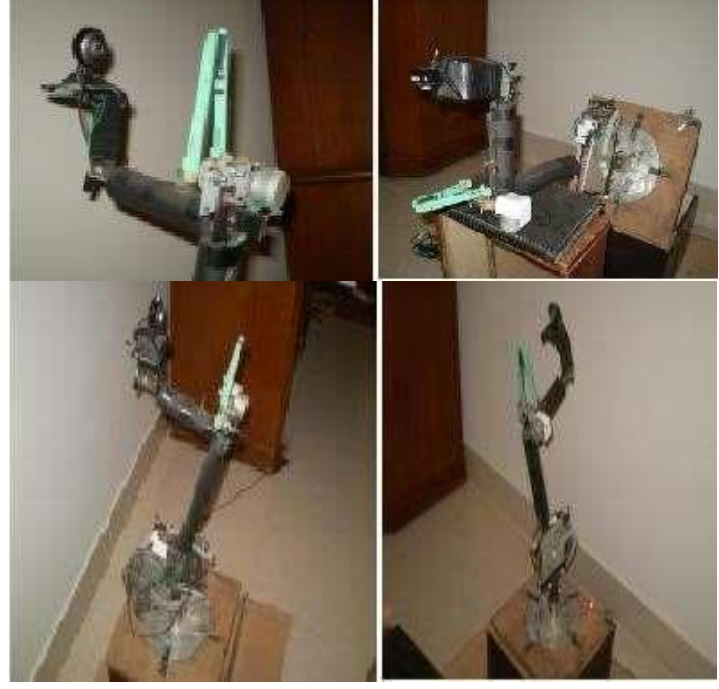

Fig. 10. Original Picture of the Prototype

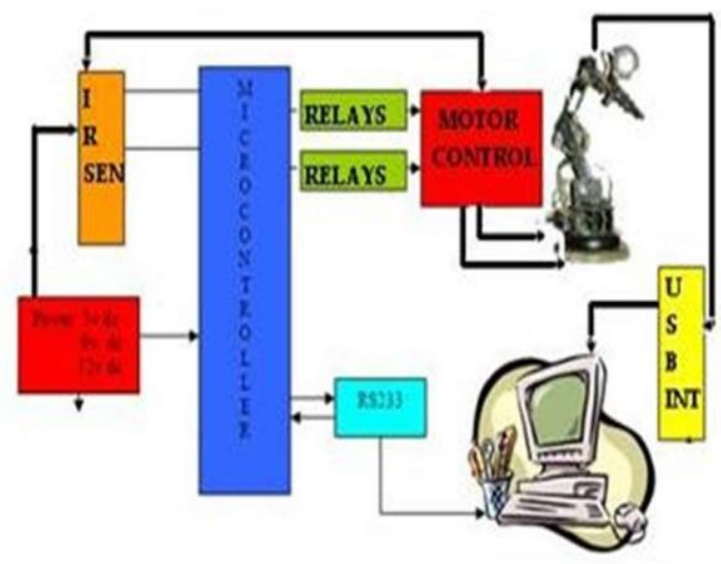

Fig. 11. Block Diagram of the Prototype

\section{CONCLUSION}

Robot successfully performed detection in various light conditions and various arbitrary objects were detected and robotic arm manipulator successfully did its placement task. The prototype has low-cost components and system is configurable for multiple movements and objects detection. Although this project is a prototype it has a vast field of future enhancement in A.I, robotics, automobile and machine learning applications.

\section{ACKNOWLEDGMENT}

Author greatly acknowledges the support and co-operation of the Prof. Dr. B.S. Chowdhary for his time and valuable suggestions also pays thanks to Robotics and Automation Research Group Department of Electronics \& Bio Medical Engineering, Mehran University of Engineering \& Technology, Pakistan.

\section{REFERENCE}

[1]. Fahad Ahmed, Haider Ali Zaidi, Syed Waqar Hussain Rizvi, Atiya Baqai and Bhawani S. Chowdhry "Electronic automatic gear shifting system for motorcycle " Communications in Computer and Information Science, 1, Volume 20, Wireless Networks, Information Processing and Systems, Pages 3-10,2009

[2]. Arsalan, M.; Aziz, A., "Low-cost Machine Vision System for dimension measurement of fast-moving conveyor products," Open Source Systems and Technologies (ICOSST), 2012 International Conference, page., no,22,27, 2022 Dec. 2012

[3]. Sokic, E.; Ahic-Djokic, M., "Simple Computer Vision System for Chess Playing Robot Manipulator as a Projectbased Learning Example," Signal Processing and Information Technology, 2008. ISSPIT 2008. IEEE International Symposium, vol., no., pp.75,79, 16-19 Dec.

[4]. Cocota, J.A.N.; Fujita, H.S.; da Silva, I.J., "A low-cost robot manipulator for education," Technologies Applied to Electronics Teaching (TAEE), 2012, vol., no., pp.164,169, 13-15 June 2012

[5]. Bulanon, D. M, and T. Kataoka. "Fruit detection system and an end effector for robotic harvesting of Fuji apples." Agricultural Engineering International: CIGR Journal 12.1 (2010). 